\title{
Adiabatic Processes in Quantum Optics
}

\author{
S. STENHOLM \\ Physics Department, Royal Institute of Technology (KTH), SCFAB \\ Roslagstullsbacken 21, 10691 Stockholm, Sweden \\ This paper reviews the use of adiabatic approximations in quantum \\ optics. The general principle is explained in terms of the Landau-Zener model \\ and the recently developed stimulated Raman adiabatic passage scheme. \\ The features characteristic of adiabatic evolution are extracted from these \\ examples. Our recent work on adiabatic level preparation and cavity mode \\ transfer of excitation is presented and discussed.
}

PACS numbers: 03.75.-b, 42.60.Da, 05.60.Gg, 03.65.Ge

\section{Adiabatic processes; an introduction}

Much of the success of quantum mechanics derives from its ability to predict energy eigenstates of various systems, ranging from elementary particles to technically important materials. These calculations are based on the stationary states and do not involve any explicit time dependence. Even the many applications to scattering phenomena reduce the situation to particle fluxes and their redistribution in the scattering region into outgoing fluxes. Only recently has it become possible to excite nonequilibrium quantum states and follow them as they evolve in time. These advances derive from the availability of short laser pulses and fast detection schemes.

In many time dependent problems, the concept of adiabaticity plays a central role. In fact, one may claim that our ability to separate a few degrees of freedom and follow their evolution derives from adiabatic separation of the various physical features involved. Some of them can be regarded as so slowly varying that they provide a stable background only.

In 1932 Landau and Zener $[1,2]$ introduced the simplest dynamical model which combines the time dependent aspect with adiabaticity and asymptotic transition probabilities. This system treats two levels which are described by the Hamiltonian 


$$
H=\left[\begin{array}{cc}
-\lambda t & V \\
V & \lambda t
\end{array}\right]
$$

For large values of time $t$, the two levels are well separated and no transitions take place. Around $t=0$ the levels cross, and population transfer becomes possible. This happens within a characteristic time scale given by

$$
T=\frac{V}{\lambda} \text {. }
$$

If $\lambda$ is small enough, this is a long time, and we may assume that an adiabatic diagonalization works. The corresponding eigenvalues are

$$
E_{ \pm}= \pm \sqrt{\left(\lambda^{2} t^{2}+V^{2}\right)}
$$

giving the solutions of the form

$$
\begin{aligned}
C(t) & \propto c_{1} \exp \left[\mathrm{i} \int_{-\infty}^{t} \mathrm{~d} t^{\prime} \sqrt{\left(\lambda^{2} t^{\prime 2}+V^{2}\right)}\right] \\
& +c_{2} \exp \left[-\mathrm{i} \int_{-\infty}^{t} \mathrm{~d} t^{\prime} \sqrt{\left(\lambda^{2} t^{\prime 2}+V^{2}\right)}\right] .
\end{aligned}
$$

In the adiabatic basis, the two incoming states go over into swapped outgoing bare states, which means that in this basis, the transition rate is the probability of not changing state; this is the general idea in adiabatic approximations. Thus in the bare state representation, the coefficient which has $C(t=-\infty)=1$, gives at $t=+\infty$ the probability to have stayed in the same bare state. The expression (1.4) does not seem to give any change of amplitude of its components, but closing the time contour in the complex plane, we notice that the contribution from the branch point at $t^{\prime}=\mathrm{i} T$ must be considered. ${ }^{*}$ This gives the result

$$
C(t=\infty) \propto \exp \left[2 \mathrm{i} \int_{0}^{\mathrm{i} T} \mathrm{~d} t^{\prime} \sqrt{\left(\lambda^{2} t^{\prime} 2+V^{2}\right)}\right]=\exp \left[-\frac{\pi V^{2}}{2 \lambda}\right] .
$$

For no coupling, $V \Rightarrow 0$, this must be unity, which serves to determine the prefator. The probability of transfer becomes thus

$$
P_{1 \rightarrow 2}=1-\exp \left[-\frac{\pi V^{2}}{\lambda}\right] \sim \pi \frac{V^{2}}{\lambda},
$$

which will be seen to agree with the result from perturbation theory. This can be obtained by an honest calculation, but its form can be found by the following outrageous argument: A naive application of the golden rule transition rate gives and "instantaneous" rate

$$
P_{1 \rightarrow 2}(t)=2 \pi V^{2} \delta(\Delta E=2 \lambda t)
$$

When this is integrated over all times, the result (1.6) emerges. Do not ask me to provide an excuse for this calculation, none can exists.

*The argument given outlines the steps only, but a detailed evaluation of the expressions in either basis gives the result given here. 
The Landau-Zener model is the prototype of all adiabatic models, it shows how quantum physics manages to transfer probabilities by using only oscillating functions. The asymptotic behaviour of scattering theory's in and out states emerges clearly. In addition, the model is exactly solvable, the asymptotic result is valid for all parameter values and physically transparent transition rates emerge. The reason the adiabatic result holds universally is that the adiabatic energies (1.3) have only one singularity in the whole complex plane. Thus there is only one time scale in the problem. The result (1.5) also shows why adiabatic results cannot be obtained in perturbation theory, $\lambda \rightarrow 0$ is an essential singularity. This also tells why it is so difficult to obtain systematic corrections to adiabatic results, no power series expansion in the slowness parameter can work.

One unpleasant feature of the Landau-Zener model is that the system cannot strictly speaking be defined at $t= \pm \infty$, because the energy levels diverge. This is corrected in the model devised by Demkov and Kunike in 1969 [3], which is also solvable, but where the adiabatic result is not exact. This has made it possible to consider the approach to adiabaticity, and in this case to evaluate the correction terms [4].

In this paper, I will discuss certain uses of adiabaticity in modern quantum optics, consider the general features of such approximations and give some examples of recent applications.

\section{The STIRAP process}

Recently an important application of adiabatic thinking has been the process called STIRAP (stimulated Raman adiabatic passage) [5, 6]. In its fundamental form, it consists of three levels, $\{|1\rangle,|2\rangle,|3\rangle\}$ coupled sequentially. However, only the middle one is supposed to be an excited state, the other two are ground states or metastables. In a rotating wave approximation, the Hamiltonian is

$$
H=\left[\begin{array}{lll}
0 & \Omega_{1} & 0 \\
\Omega_{1} & \Delta & \Omega_{2} \\
0 & \Omega_{2} & 0
\end{array}\right]
$$

The intermediate level detuning $\Delta$ may differ from zero, and the coupling rates $\Omega_{i}$ are taken to be time dependent. If we assume that these are slowly varying, we perform an instantaneous diagonalization by using the states

$$
\begin{aligned}
& \left|\varphi_{+}\right\rangle=\sin \varphi \sin \theta|1\rangle+\cos \varphi|2\rangle+\sin \varphi \cos \theta|3\rangle, \\
& \left|\varphi_{0}\right\rangle=\cos \theta|1\rangle-\sin \theta|3\rangle, \\
& \left|\varphi_{-}\right\rangle=\cos \varphi \sin \theta|1\rangle-\sin \varphi|2\rangle+\cos \varphi \cos \theta|3\rangle,
\end{aligned}
$$

where we have the eigenvalues $\left\{w_{+}, 0, w_{-}\right\}$with

$$
w_{ \pm}=\frac{1}{2}\left(\Delta \pm \sqrt{\Delta^{2}+4 \Omega_{0}^{2}}\right)
$$


and

$$
\Omega_{0}^{2}=\Omega_{1}^{2}+\Omega_{2}^{2}
$$

The angles are defined by

$$
\tan \theta=\frac{\Omega_{1}}{\Omega_{2}}, \quad \tan 2 \varphi=\frac{2 \Omega_{0}}{\Delta} .
$$

For $\Delta=0$, we can choose $\sin \varphi=\cos \varphi=1 / \sqrt{2}$.

The state

$$
\left|\varphi_{0}\right\rangle=\frac{\Omega_{2}|1\rangle-\Omega_{1}|3\rangle}{\sqrt{\Omega_{1}^{2}+\Omega_{2}^{2}}}
$$

is seen to belong to a zero eigenvalue. If we now let

$$
\lim _{t \rightarrow-\infty} \frac{\Omega_{1}}{\Omega_{2}}=0, \quad \lim _{t \rightarrow+\infty} \frac{\Omega_{1}}{\Omega_{2}}=\infty,
$$

we find that starting in the state $|1\rangle$, the transfer to the state $|3\rangle$ is perfect

$$
\lim _{t=-\infty}\left|\varphi_{0}\right\rangle=|1\rangle, \quad \lim _{t=+\infty}\left|\varphi_{0}\right\rangle=-|3\rangle
$$

As this approach starts by coupling two totally empty states it is usually termed "counter-intuitive".

Using the states (2.2) as basis states, the Hamiltonian becomes

$$
H=\left[\begin{array}{ccc}
+w & 0 & 0 \\
0 & 0 & 0 \\
0 & 0 & -w
\end{array}\right]+\left[\begin{array}{ccc}
0 & \mathrm{i} \dot{\theta} \sin \varphi & \mathrm{i} \dot{\varphi} \\
-\mathrm{i} \dot{\theta} \sin \varphi & 0 & -\mathrm{i} \dot{\theta} \cos \varphi \\
-\mathrm{i} \dot{\varphi} & \mathrm{i} \dot{\theta} \cos \varphi & 0
\end{array}\right] .
$$

The second term is neglected in the adiabatic approximation. The dynamic evolution is confined entirely to the subspace with vanishing eigenvalue. Thus the adiabatic limit effects the population transfer $|1\rangle \rightarrow|3\rangle$ without populating the excited state $|2\rangle$.

This STIRAP process has been applied extensively to laser-induced processes, and many experimental realizations have already been achieved [7]. It provides another efficient prototype of adiabatic transfer in addition to the LandauZener situation. It illustrates some fundamental features of all such processes, which we can summarize as follows:

- If the interaction is not analytic in time, the adiabatic behaviour is dominated by the discontinuity. If this occurs in the $\alpha$-th derivative, and the adiabatic time scale is given by $T$, the corrections to the adiabatic result disappears like $T^{-(\alpha+1)}$ as found in Ref. [8]; see also [9].

- For smooth pulses, the analytic behaviour is strongly dependent on how the couplings behave at infinite times, but the corrections to adiabaticity disappear like $\exp (-\beta T)$, where $\beta$ is determined by the details of the interactions [4]. In most numerical work the pulses are supposed to have a Gaussian form. From the point of view of adiabaticity, this may not be optimal; simple hyperbolic dependence may be more advantageous. 
In order to illustrate the adiabatic features of the problem, we consider a somewhat modified model of the STIRAP situation. As the initial pulse $\Omega_{2}(t)$ couples only empty levels for $t \sim-\infty$ we can let it start at a fixed value without loss of physics. The same holds for the other pulse $\Omega_{1}(t)$; at large times it ideally couples only empty levels and can approach a constant value. Thus we may arrange the time dependences such that $\Omega_{0}$ in (2.4) is a constant, which grossly simplifies the treatment. In Ref. [9] we show that the choice

$$
\Omega_{1}^{2}=\frac{\Lambda^{2}}{1+\exp (-t / T)}, \quad \Omega_{2}^{2}=\frac{A^{2}}{1+\exp (t / T)}
$$

allows an analytic solution for the population on the final state in the form

$$
\left|c_{3}(\infty)\right|^{2}=\tanh ^{4}(\pi \Lambda T) \sim 1-8 \exp (-2 \pi \Lambda T),
$$

where the expansion holds in the adiabatic limit $T \rightarrow \infty$. In the perturbative limit, $\Lambda \rightarrow 0$, the process is a two-step transition and its probability properly goes as $(A T)^{4}$. The exponential dependence characteristic of adiabatic behaviour is seen to follow all the way to final full adiabaticity.

In our work [9], we investigated the non adiabatic corrections to a STIRAP process with pulsed couplings. We were indeed able to find the expected exponential behaviour over four orders of magnitude in the adiabaticity time scale. However, for very large values of $T$, the corrections start to oscillate violently. This feature is not explained within the existing theory but it is found to be a persistent feature of such systems. In an analytically solvable model of the STIRAP, Vitanov and Stenholm [10] managed to obtain an adiabatic result of the type

$$
1-\left|c_{3}(\infty)\right|^{2} \sim A \exp (-\pi \Lambda T)+\frac{B}{1+(\Lambda T)^{2}} \cos ^{2} \varphi(\Lambda T),
$$

where $A$ and $B$ are constants and $\varphi(A T)$ is a given function. This result displays the exponential behaviour characteristic of the adiabatic situation, but also the asymptotically dominating oscillatory contributions. We know of no simple approximative way to extract these terms in the general case. For the details the reference should be consulted.

\section{Features of adiabatic evolution}

The Schrödinger equation we consider is assumed to scale as follows:

$$
\mathrm{i} \frac{\partial \Psi}{\partial t}=\Lambda H(t / T) \Psi
$$

where the parameters $A$ and $T$ denote the strength and the duration of the interaction. By introducing the scaled time $\tau=t / T$, we can write the equation as

$$
\mathrm{i} \frac{\partial \Psi}{\partial \tau}=\Lambda T H(\tau) \Psi
$$


which shows that the adiabatic limit $T \rightarrow \infty$ is equivalent with the strong coupling limit $\Lambda \rightarrow \infty$. This has been utilized frequently in our numerical calculations, when we do not want to change the coding of the interaction interval in time.

If we take the rate of change of the Hamiltonian to be formally given by

$$
\frac{1}{T}=\frac{\dot{H}}{H},
$$

we find the condition for adiabaticity to be

$$
\hbar \frac{\dot{H}}{H} \ll H .
$$

If all time dependence resides in the off-diagonal coupling $V$ and the energy eigenvalues are spaced by $\Delta E$ this relation gives

$$
\hbar \frac{\dot{V}}{V} \ll \Delta E .
$$

This is the form given in most texts. In the case (3.1) the condition becomes

$$
\Lambda T \gg 1
$$

as we found above; this is the form usually given for adiabatic following in magnetic resonance [11].

In the STIRAP situation, the Hamiltonian has the property

$$
\lim _{t \rightarrow \pm \infty} H=\lim _{t \rightarrow \pm \infty}\left[\begin{array}{ccc}
0 & \Omega_{1} & 0 \\
\Omega_{1} & 0 & \Omega_{2} \\
0 & \Omega_{2} & 0
\end{array}\right]=0 .
$$

In this case, the right-hand side of (3.4) goes to zero and the inequality (3.4) cannot remain valid for $t \rightarrow \pm \infty$. It thus seems that the initial and final times are not adiabatic, but for large ranges of the interaction parameter strength, the adiabatic transfer dominates and exponential corrections only ensue. The oscillatory behaviour must thus derive from the initial and final periods when adiabaticity is violated.

For the moment we do not know any analytic approach to Hamiltonians which vanish asymptotically at $t= \pm \infty$. This singularity prevents us from closing the contours in the complex time plane. The fully adiabatic behaviour found in Eq. (2.11) derives from the fact that, in this model, the Hamiltonian never goes to zero, but due to the condition $\Omega_{0}^{2}=\Omega_{1}^{2}+\Omega_{2}^{2}=$ constant, the eigenenergies never vanish and adiabaticity can be made to hold at all times. This feature is common in many adiabatic models; the simple STIRAP is unusual in this aspect. Even here, more involved level schemes, however, do tend to retain some terms in the Hamiltonian finite. 


\section{Applications}

\subsection{Coherent preparation}

We consider a generalization of the simple three-level STIRAP to a situation with $N$ levels $\{|1\rangle,|2\rangle, \ldots|N\rangle\}$. The coupling is assumed to be of simple star-type with the level $|N\rangle$ coupled to all the other ones, but these are not coupled among themselves. The state $|N\rangle$ is assumed to be an excited one, and it may be able to decay spontaneously. If the states coupled to $|N\rangle$ belong to the same angular momentum manifold, the dipole selection rules state that $N$ can be at most four, but we may safely assume it to be possible to combine several different manifolds. The coherence necessary in the couplings may well be achieved with coherent laser sources.

If we assume the initial state to be

$$
|\Psi(t=-\infty)\rangle=|1\rangle
$$

and try to prepare a preassigned linear combination

$$
|\Psi(t=\infty)\rangle=c_{2}|2\rangle+c_{3}|3\rangle+\ldots c_{N-1}|N-1\rangle,
$$

we may use a generalization of the STIRAP approach. This has been shown to be possible by straightforward computations [12], and an application of a coherent control algorithm [13]. The amusing conclusion of the latter approach is that the program can pick out the counter-intuitive pulse sequence on its own as the optimal path to the desired final state.

If we introduce the transformation $U$ which diagonalizes the Hamiltonian

$$
D=U H U^{\dagger},
$$

we find after changing to the adiabatic basis the Hamiltonian of the form

$$
H=D-\mathrm{i} U^{\dagger} \frac{\partial}{\partial t} U \approx\left[\begin{array}{cccc}
0 & \mathrm{i} \boldsymbol{\Gamma} & & \cdot \\
-\mathrm{i} \boldsymbol{\Gamma}^{\dagger} & \mathbf{0} & & \cdot \\
\cdot & \cdot & {\left[\begin{array}{cc}
\Omega & 0 \\
0 & -\Omega
\end{array}\right]}
\end{array}\right]
$$

The dots indicate nonadiabatic coupling terms which may be neglected. The strongly coupled subpace with eigenvalues $\pm \Omega$ is called a "bright subspace" because it contains an admixture of the upper state which may emit a photon spontaneously. The remaining $N-2$ states belong to zero eigenvalues and do not contain any admixture of the single excited state. The operator $\boldsymbol{\Gamma}$ gives the adiabatic corrections in this subspace, and in spite of the fact that it is assumed small in the proper limit, it cannot be neglected. The reason is that in this subspace the diagonal elements are zero, and thus the condition (3.5) cannot be satisfied. In this dark subspace, the levels are easily mixed and hence the influence is dominated by $\boldsymbol{\Gamma}$. On the other hand, the dynamic evolution takes place almost entirely in 
this subspace, and thus we have reduced the original $N$-dimensional problem to one of evolution in a subspace of $N-2$ dimensions with the effective Hamiltonian

$$
H_{\mathrm{eff}}=\left[\begin{array}{cc}
0 & \mathrm{i} \boldsymbol{\Gamma} \\
-\mathrm{i} \boldsymbol{\Gamma}^{\dagger} & \mathbf{0}
\end{array}\right]
$$

This shows how separation of fast and slow time scales can provide an effective Hamiltonian description in a reduced Hilbert space where the fast subspace has been projected away.

Then one may ask the question whether it is possible to affect a desired transformation in the full $N$-dimensional space by only dynamics in the subspace of slow adiabatic evolution. In Ref. [14] we show in the framework of a solvable model that such a process is possible. We do not claim that an arbitrary transformation can be achieved this way, but in many situations it can provide a solution.

\subsection{Cavity $Q E D$}

A simplified model of cavity quantum electrodynamics (QED) can be provided by the Jaynes-Cummings model. In this section we are going to use a version with two cavity modes interacting with a single two-level atom. The Hamiltonian is given by

$$
\begin{aligned}
H= & \frac{\Omega}{2}\left(\sigma_{3}+1\right)+\omega\left(a_{1}^{\dagger} a_{1}+a_{2}^{\dagger} a_{2}\right) \\
& +g_{1}\left(a_{1}^{\dagger} \sigma^{-}+a_{1} \sigma^{+}\right)+g_{2}\left(a_{2}^{\dagger} \sigma^{-}+a_{2} \sigma^{+}\right),
\end{aligned}
$$

where $a_{i}(i=1,2)$ are the bosonic mode annihilation operators, and the $\sigma^{+}$and $\sigma^{-}$are the ordinary Pauli operators. We here assume that all frequencies are the same, $\Omega=\omega$; the atomic frequency could be different without any change in the treatment below.

If we now introduce the operators

$$
B=\frac{1}{\sqrt{g_{1}^{2}+g_{2}^{2}}}\left(g_{1} a_{1}+g_{2} a_{2}\right)
$$

and

$$
A=\frac{1}{\sqrt{g_{1}^{2}+g_{2}^{2}}}\left(g_{2} a_{1}-g_{1} a_{2}\right),
$$

we find that they obey bosonic commutation relations and the Hamiltonian (4.6) can be written as

$$
H=\frac{\omega}{2}\left(\sigma_{3}+1\right)+\omega\left(A^{\dagger} A+B^{\dagger} B\right)+G\left(B \sigma^{+}+B^{\dagger} \sigma^{-}\right),
$$

where the new coupling constant is

$$
G=\sqrt{g_{1}^{2}+g_{2}^{2}} .
$$


This Hamiltonian corresponds to the ordinary single mode case, with the oscillator mode $A$ totally decoupled from the atom. This can consequently be solved directly as in the single mode case [15]. The model may, however, also be used in a different manner [16]. relations

If we can control the coupling constants in such a way that they satisfy the

$$
\lim _{t \rightarrow-\infty}\left(\frac{g_{1}}{g_{2}}\right)=0, \quad \lim _{t \rightarrow+\infty}\left(\frac{g_{1}}{g_{2}}\right)=-\infty,
$$

we find for an arbitrary photon state of the form

$$
\begin{aligned}
& \lim _{t \rightarrow-\infty}|f\rangle \equiv \lim _{t \rightarrow-\infty} f\left(A^{\dagger}\right)|0\rangle=f\left(a_{1}^{\dagger}\right)|0\rangle, \\
& \lim _{t \rightarrow+\infty}|f\rangle \equiv \lim _{t \rightarrow+\infty} f\left(A^{\dagger}\right)|0\rangle=f\left(a_{2}^{\dagger}\right)|0\rangle .
\end{aligned}
$$

Thus we see that this adiabatic change of the couplings can transfer any arbitrary quantum state in one cavity mode into the other one; we do not need to know the state in advance. The process is a direct generalization of the STIRAP situation, the couplings are turned on in a similar counter-intuitive way, and the result is analogous. The larger energies that are involved, the higher the basis states we want to transfer, the more demanding we have found the requirement of adiabaticity, but the desired result is always achieved when the process is slowed down or equivalently the coupling strength is increased.

The scheme explained above does require that the two cavity modes involved are fully degenerate. It is, however, possible to find a way to overcome the restriction $\omega_{1}=\omega_{2}$. We then start with the Hamiltonian

$$
\begin{aligned}
H= & \frac{\Omega}{2}\left(\sigma_{3}+1\right)+\omega_{1} a_{1}^{\dagger} a_{1}+\omega_{2} a_{2}^{\dagger} a_{2} \\
& +g_{1}\left(a_{1}^{\dagger} \sigma^{-}+a_{1} \sigma^{+}\right)+g_{2}\left(a_{2}^{\dagger} \sigma^{-}+a_{2} \sigma^{+}\right) .
\end{aligned}
$$

We assume that we find a way to modulate the coupling constants to be given by

$$
g_{i}=2 \lambda_{i} \cos \Delta t, \quad(i=1,2)
$$

where

$$
\Delta=\frac{\omega_{2}-\omega_{1}}{2} .
$$

On this time dependent Hamiltonian, we apply the unitary transformation

$$
U=\exp \left[\mathrm{i} \Delta\left(a_{1}^{\dagger} a_{1}-a_{2}^{\dagger} a_{2}\right) t\right] .
$$

The rotating-wave-approximation is assumed to hold, giving

$$
2 \cos \Delta t \times \exp ( \pm \mathrm{i} \Delta t) \sim 1,
$$

which produces the transformed Hamiltonian 


$$
\begin{aligned}
\widetilde{H}= & U^{\dagger} H U-\mathrm{i} U^{\dagger} \frac{\partial}{\partial t} U=\frac{\Omega}{2}\left(\sigma_{3}+1\right)+\left(\omega_{1}+\Delta\right) a_{1}^{\dagger} a_{1}+\left(\omega_{2}-\Delta\right) a_{2}^{\dagger} a_{2} \\
& +\lambda_{1}\left(a_{1}^{\dagger} \sigma^{-}+a_{1} \sigma^{+}\right)+\lambda_{2}\left(a_{2}^{\dagger} \sigma^{-}+a_{2} \sigma^{+}\right) .
\end{aligned}
$$

Now the modes have the same frequency $\frac{1}{2}\left(\omega_{2}+\omega_{1}\right)$ and we are in the previously described situation. The correction terms oscillate as $\exp ( \pm 2 \mathrm{i} \Delta t)$, and their influence is expected to be of the order $(\lambda / \Delta)^{2}$.

The adiabaticity condition requires that $\lambda T \gg 1$, which implies that also $\Delta T \gg 1$. This puts restrictions on the method, but in the opposite limit, $\Delta T \ll 1$, the detuning is not necessarily very efficient in destroying the adiabaticity. This situation with different mode frequencies has not been investigated numerically.

The adiabatic transfer takes place in the frame defined by the unitary transformation (4.16). Because however all moments of the form

$$
\left\langle\left(a_{1}^{\dagger} a_{1}\right)^{n}\left(a_{2}^{\dagger} a_{2}\right)^{m}\right\rangle=\left\langle U\left(a_{1}^{\dagger} a_{1}\right)^{n}\left(a_{2}^{\dagger} a_{2}\right)^{m} U^{\dagger}\right\rangle
$$

have the same values in both frames, transfer of field population in one frame, will appear as the same transfer in the other one. Only coherences between the amplitudes will have a different time evolution. This is, however, rather easily taken into account in the present case.

\section{Conclusion}

This paper gives a short review of some of the interesting and fundamental aspects of adiabatic processes occurring in quantum optics systems. Some have found wide applicability in laser spectroscopy of atoms and molecules, others remain to be introduced into experimental situations. In this concluding section, I summarize some of the main points of the present text:

- Adiabatic processes are both efficient and robust when they can be implemented. In many cases, the adiabatic limit can be reached when the coupling strength is increased.

- The corrections to adiabatic transfer of population tend to vanish exponentially with the adiabaticity parameter.

- In systems like STIRAP, where the Hamiltonian becomes exactly zero at some limiting times, the adiabaticity breaks down, and deviations from exponential behaviour emerges. This may manifest itself as oscillatory dependences on the adiabaticity parameter. These are genuine features of such systems, but they do manifest themselves at such low levels of corrections, that they may lack practical significance.

- The original adiabatic processes have been chosen such that the energy is conserved between initial and final states. This is, however, not necessary; some generalizations of STIRAP do not have this feature. If one wants to 
compensate for energy mismatch, the method introduced in Sec. 4.2 for cavity modes can be applied. Here energy differences are compensated by equipping the levels involved with suitably generated side-bands. In the suggested form, or suitably generalized, the method can be applied to most situations of adiabatic transfer.

- Finally we stress that adiabatic methods are very common and efficient when they are applicable. We do not, however, know of a systematic way to generate approximations, where the lowest order one is the well-known adiabatic result, and there exists a well-defined method to generate higher order corrections.

\section{Acknowledgments}

I have had the pleasure to work on these problems with many collaborators. I owe them all thanks for the results we have obtained and the many discussions we have enjoyed. I especially want to mention: T.A. Laine, B.M. Garraway, K.-A. Suominen, N.V. Vitanov, F. Renzoni, Z. Kis, F. Mattinson, and M. Kira.

\section{References}

[1] L.D. Landau, Phys. Z. Sowjetunion 2, 46 (1932).

[2] C. Zener, Proc. R. Soc. Lond. A 137, 696 (1932).

[3] Yu. Demkov, M. Kunike, Vestn. Leningr. Univ. Ser. Fiz. Khim 16, 39 (1969).

[4] K.-A. Suominen, B.M. Garraway, S. Stenholm, Opt. Commun. 82, 260 (1991).

[5] J. Oreg, F.T. Hioe, J.H. Eberly, Phys. Rev. A 29, 690 (1984).

[6] J.R. Kuklinski, U. Gaubatz, F.T. Hioe, K. Bergmann, Phys.Rev. A 40, 6741 (1989); B.W. Shore, K. Bergmann, J. Oreg, S. Rosenwaks, Phys. Rev. A 44, 7442 (1991); B.W. Shore, K. Bergmann, A. Kuhn, S. Schiemann, J. Oreg, Phys. Rev. A 45, 5297 (1992).

[7] K. Bergmann, H. Theuer, B.W. Shore, Rev. Mod. Phys. 70, 1003 (1998).

[8] M.V. Berry, J. Phys. A 15, 3693 (1982).

[9] T.A. Laine, S. Stenholm, Phys. Rev. A 53, 2501 (1996).

[10] N.V. Vitanov, S. Stenholm, Opt. Commun. 127, 215 (1996).

[11] A. Abragam, The Principles of Nuclear Magnetism, Oxford University Press, Oxford 1961, p. 66.

[12] N.V. Vitanov, K.-A. Suominen, B.W. Shore, J. Phys. B, At. Mol. Opt. Phys. 32, 4535 (1999); F. Renzoni, S. Stenholm, Opt. Commun. 189, 69 (2001).

[13] Z. Kis, S. Stenholm, J. Mod. Opt. 49, 111 (2002).

[14] Z. Kis, S. Stenholm, Phys. Rev. A 64, 063406-1 (2001).

[15] S.M. Dutra, P.L. Knight, H. MoyaCessa, Phys. Rev. A 48, 3168 (1994); S.M. Dutra, P.L. Knight, Phys. Rev. A 49, 1506 (1994).

[16] F. Mattinson, M. Kira, S. Stenholm, J. Mod. Phys. 48, 889 (2001). 